\title{
Determination of the Pellets Parameters by Image Processing Methods
}

\author{
Teddy Craciunescu ${ }^{\mathrm{a}}$, Peter Lang ${ }^{\mathrm{b}}$, Andrea Murari ${ }^{\mathrm{c}}$, Tamás Szepesi ${ }^{\mathrm{d}}$, Sándor Kálvin ${ }^{\mathrm{d}}$, Gábor Kocsis ${ }^{\mathrm{d}}$, \\ Arturo Alonso $^{\mathrm{e}}$, Ion Tiseanu ${ }^{\mathrm{a}}$, Vasile Zoita ${ }^{\mathrm{a}}$ and JET EFDA Contributors ${ }^{\S}$
}

JET-EFDA Culham Science Centre, Abingdon, OX14 3DB, UK.

${ }^{a}$ EURATOM-MEdC Association, Institute for Laser, Plasma and Radiation Physics, Bucharest, Romania

${ }^{b}$ Association EURATOM-IPP, Max-Planck-Institut für Plasmaphysik, Garching, Germany

${ }^{c}$ Consorzio RFX, Associazione EURATOM-ENEA per la Fusione, Padova, Italy

${ }^{d}$ RMKI KFKI, EURATOM Association, Budapest, Hungary

${ }^{\mathrm{e}}$ Laboratorio Nacional de Fusion, EURATOM-CIEMAT, Madrid, Spain

Injection of solid, cryogenic hydrogen isotope pellets in tokamaks is used for particle fuelling as well as for ELM control. The efficiency depends on technical control variables such as pellet size, velocity, frequency and poloidal launch position. Recently developed image processing methods have been improved and adapted in order to evaluate some of these key parameters. An optical flow method has been used for the determination of the ice extrusion velocity based on the image sequences provided by a CCD camera viewing the ice at the exit of the nozzles of the extrusion cryostat. The reconstruction of pellet volume has been performed using images provided by another CCD camera, coupled to a set of optical barriers used for pellet velocity measurement. A Bayesian statistical analysis has been applied, calculating the probability distribution function of the pellet volume based on three measured parameters of the pellet shadow: area, smallest dimension, largest dimension.

Keywords: Pellets parameters, Image processing, Optic flow, Shadowgraphy

\section{Introduction}

Injection of solid, cryogenic hydrogen isotope pellets in tokamaks is used for particle fuelling as well as for ELM control (triggering and mitigation). The method has been demonstrated to open access to operational regimes not reachable by gas puffing [1]. Pellet fuelling in the high confinement (H-mode) regime is characterized by the curvature induced drift of the high pressure plasmoid forming around the ablating pellet [2]. This can be exploited in order to improve the fuelling efficiency by launching pellets from the magnetic high field side (HFS) [3]. The pellets can also be used for the control of edge localised modes (ELMs) [4]. ELM triggering by pellets has been recognised as a potentially useful tool to mitigate type-I ELMs in large fusion experiments [5]. However, a controlled high fuelling efficiency is needed as otherwise the beneficial effects are spoiled by the increase of neutral pressure from fuel losses. ELM triggering and the variation of the ELMs dynamics depend on technical control variables such as pellet size, velocity, frequency and poloidal launch position.

A recently developed optical flow (OF) method [6] was adapted in order to evaluate the ice extrusion velocity based on the image sequences provided by a CCD camera viewing the ice at the exit of the nozzles of the extrusion cryostat [7]. The method combines the advantages of local methods (robust under noise) and global techniques (which yield dense flow fields). Several image processing techniques are used in order to reduce the computing time ensuring at the same time a good quality of the $\mathrm{OF}$ evaluation. A technique able to prevent the calculation of an inaccurate velocity was implemented.

The reconstruction of pellet volume has been performed using images provided by another CCD camera, coupled to a set of optical barriers used for pellet velocity measurement. This camera was installed on a diagnostic chamber located at the injector exit of the JET high frequency pellet injector (see again Ref. 7), providing images about the flying pellet. A Bayesian statistical analysis is applied, calculating the probability distribution function of the pellet volume based on three measured parameters of the pellet shadow: area, smallest dimension, largest dimension. The only assumption made in the algorithm is the presumed cylindrical shape of the pellets. The advantage of the method is that beside the pellet volume, its error bars can also be estimated.

\section{Determination of Ice Extrusion Velocity by Optical Flow Method}

One of the main challenges in computer vision is the automatic extraction of motion information from image sequences. Without any prior knowledge about the captured scene, the OF approach attempts to retrieve the direction and velocity of travelling objects. The estimation of motion information from image sequences assumes the brightness constancy constraint:

$$
f(x+\Delta x, y+\Delta y, t+1)-f(x, y, t)=0,
$$

\footnotetext{
* Corrsponding author E-mail: teddy.craciunescu@jet.uk

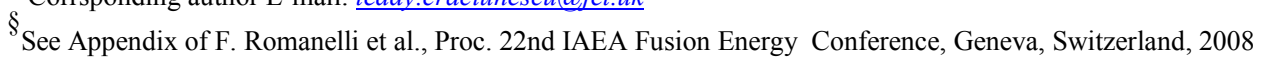


where $f$ is the intensity and $\Delta x$ and $\Delta y$ are the two displacements from one frame to the next. As the displacements are small, using the Taylor expansion, the OF constraint (1) can be reformulated:

$$
f_{x} u+f_{y} v+f_{t}=0
$$

where subscripts denote partial derivatives and $u$ and $v$ are the two components of OF. From the mathematical point of view, the problem (2) is an ill-posed one. In order to deal with the aperture problem, the Lucas-Kanade approach assumes that the unknown OF vector is constant within a neighbourhood of size $\rho$ [8]. Therefore $u$ and $v$ can be determined at the location $(x, y, t)$ from a weighted least square fit by minimising the function:

$$
E_{L K}(u, v)=K_{\sigma} *\left(\left(f_{x} u+f_{y} v+f_{t}\right)^{2}\right)
$$

where $K_{\sigma}(x, y)$ is a Guassian smoothing operator which is used in order to remove noise and to stabilize the differentiation process. A sufficiently large value for $\rho$ is very successful in rendering the method robust against noise. However the problem remains severe in flat regions of the emission, where the image gradient vanishes and, consequently, the method is unable to produce dense flow fields. In order to avoid this drawback, Bruhn et al. [9] suggested the introduction of a Horn-Schunk type [10] supplementary regularizing smoothness term. Therefore the optical flow $(u, v)$ is determined as the minimizer of the following functional:

$$
E_{H S}(w)=\int_{\Omega}\left(w^{T} J_{\rho}\left(\nabla_{3} f\right) w+\alpha|\nabla w|^{2}\right) d x d y
$$

where $J_{\rho}\left(\nabla_{3} f\right)$ is the structure tensor [11] and $\alpha>$ Odetermines the amount of smoothness.

This class of methods have been studied and optimized for the very specific case of JET images. The methods have been already used for the evaluation of the speed of various plasma instabilities, in particular ELM filaments and MARFEs (see Refs. 6 and 12). The OF method is now further adapted for the determination of the ice extrusion velocity.

In order to be able to deal with relatively large displacements of objects between consecutive frames we have used a multi-resolution coarse-to-fine procedure. A pyramid of multi resolution images is derived from the original frame by successive down-sampling and Gaussian smoothing steps. OF calculation starts at the coarse level, where the displacements are small and consequently the linearization of the grey value constancy assumption is satisfied. This estimate is then refined step by step along the pyramidal structure. The pyramidal structure has been optimized in order to minimize the total computing time ensuring in the same time a proper evaluation of the optical flow. A number of 6 levels in the multi-resolution pyramid are able to deal with the displacements existing in the images recorded by the CCD camera.
A significant reduction of the computing time can be achieved by image pre-processing techniques which consist in object detection and tracking. The region of interest (ROI) is narrowed to the area where objects in the image are moving. As the ribbons of ice are floating in the image (see. Fig. 1) ROI must be estimated for each pair of frames. The difference between two consecutive frames can be used to highlight moving objects in the image (Fig. 2 - left). However, due to noise in the initial images $I_{1}, I_{2}$, the image difference $I d=I_{1}-I_{2}$ is affected also by noise which does not allow the automatic delimitation of ROI. This noise can be removed by appropriate image segmentation. We used histogram-based segmentation methods. They are very efficient when compared to other image segmentation methods because they typically require only one pass through the pixels. The $I_{d}$ gray-level histogram is a highly skewed distribution with high peak values. Therefore efficient calculations are provided by a modified triangle algorithm [13] which assumes a maximum peak towards one end of the gray-level histogram. This method provides a fast solution. Comparable results, in what concerns the quality of the segmentation have been obtained using the more sophisticated method of Huang et al. [14] which is based on minimizing the measures of fuzziness of the image. The method uses entropy as the measure of fuzziness. The image pixel membership functions are dependent of the threshold value and they reflect the distribution of pixels values in two classes, thus this method minimizes the classification error. After segmentation of $I_{d}$, a ROI can be defined (Fig. 2 - right). In most cases, using this technique, the size of the images which constitutes the input for the OF calculations is reduced with a factor of 4 .

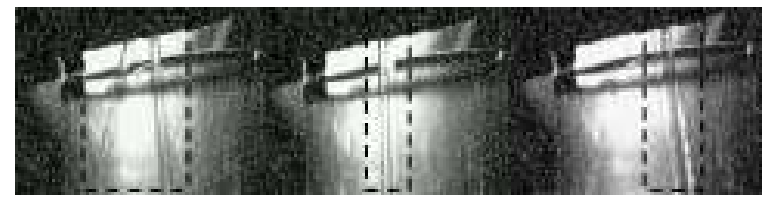

Figure 1 - Three different frames from the image sequence showing the extruded deuterium ice in case of JET pulse \#76379. ROI is represented on the images (dashed contour).

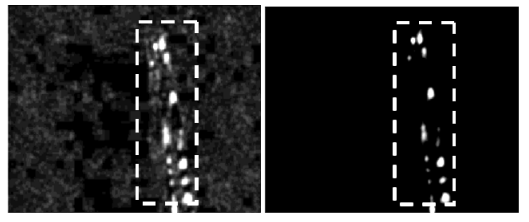

Figure 2 -Image difference $I_{d}$ of frames 1784 and 1785 (left) and $\mathrm{I}_{\mathrm{d}}$ after thresholding. ROI is represented on the images (dashed contour).

Using the above described techniques, the total OF image processing time is $10 \mathrm{~s}$ per pair of frames, allowing the analysis of large amount of video data, corresponding to a specific shot, in reasonable time.

A representative result concerning the evaluation of the ice extrusion velocity is presented in Fig. 3. Due to the strong saturation of the images provided by the video camera (Fig. 3 top-left), the ice ribbon-structure is only partially 
visible. Therefore the speed velocity field (Fig. 3 top-right) is reconstructed only for the visible ribbon structure.

The width of the ice ribbon $(1.2 \mathrm{~mm})$ was used to convert pixels in length units. The optical flow is determined using consecutive frames $\left(I_{1}, I_{2}\right)$ from a sequence of images. Using $I_{1}$ and the calculated OF, a version $I_{2-\text { rec }}$ of $I_{2}$ can be reconstructed. The similarity between $I_{2}$ and $I_{2-\text { rec }}$ can be used in order to assess the accuracy of the OF calculations.

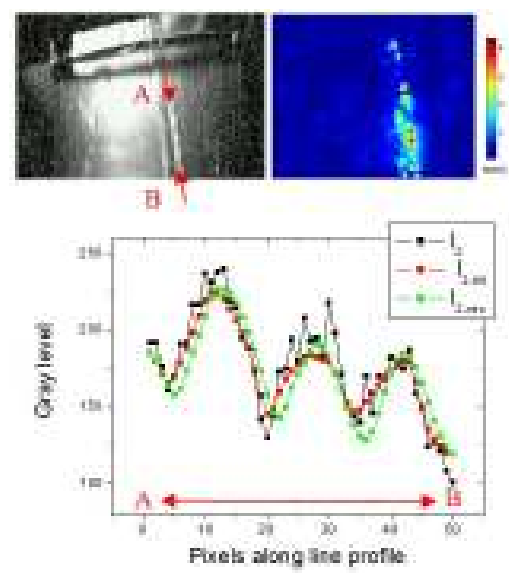

Figure 3 - Illustration of OF calculations: frame 1784 (left), calculated velocity field (top-right) and line profiles through the images $\mathrm{I}_{2}$ and its reconstruction $\mathrm{I}_{2 \text {-rec }}$ (bottom).

Line profiles through images $I_{2}$ and $I_{2 \text {-rec }}$, along the direction $\mathrm{AB}$, characterized by significant $\mathrm{OF}$ values, are presented in also in Fig. 3. For the OF velocity speed calculation, Gaussian smoothing is applied for the input images. Therefore line profile through $I_{2-\text { rec }}$ should be compared with the line profile through $I_{2-\text { filt }}$, obtained after Gaussian filtering of $I_{2}$. The degree of similarity between $I_{2}$ and $I_{2-\text { rec }}$ has been used, as a routine procedure, for the assessment of the quality of OF evaluation. This criterion can detect if the basic assumptions of the OF model may not be verified, due to e.g. image saturation, discontinuous movement, reshaping of image objects. It represents an efficient and reliable way to prevent inaccurate evaluation of the velocity field.

\section{Determining Pellet Volume Using Dual-View Shadowgraphy Diagnostic and Bayesian Statistics}

The shadowgraphy diagnostic is a powerful tool for the invivo inspection of high-speed pellets. Pellets are usually driven to the plasma in closed guiding tubes, making it impossible to observe them. In the shadowgraphy system, the guiding tube is opened for a short distance, that is, the pellet passes through a free-flight region where the pellet can be observed. For the JET shadowgraphy system the length of this free-flight region is $\sim 20 \mathrm{~mm}$. The system consists of a digital camera and a pulsed laser, acting as a very short $(\sim 1 \mu \mathrm{s})$ and intense flash (see Fig. 4). The illumination of the pellet produces a very high-contrast shadow of the pellet, and the recorded image is sharp even for pellet velocities in the $100-200 \mathrm{~m} / \mathrm{s}$ range. At JET the shadowgraphy diagnostic has two views, that is, the pellet can be observed from two directions, $90^{\circ}$ apart, at the same time. This setup theoretically allows for a better reconstruction of the pellet volume, compared to a singleview system, like the one at ASDEX Upgrade (AUG) [15].

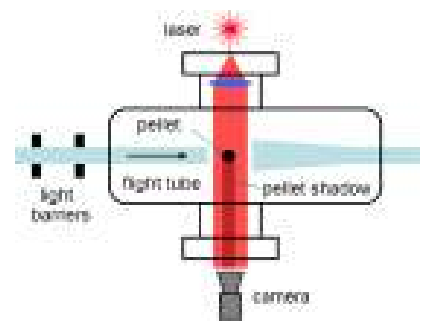

Figure 4 - Setup of the shadowgraphy diagnostic

For the reconstruction of the volume for an arbitrary threedimensional object neither one nor two views are sufficient. However, if the 3D object has some symmetries, even a single view can provide a reasonable estimate, as demonstrated for the AUG system (see again Ref. 15). Both in the AUG and JET case one can assume the pellets to be cylindrical, a highly symmetric form, having only three parameters that determine the shape of the shadow: pellet radius $(r)$, pellet height $(h)$ and angle $(\alpha)$ between the camera sight direction and the pellet's orientation (the direction of the surface vector of the pellet's circular base). These three parameters exactly define the 'barrel' shaped shadow of the pellet. In the reconstruction algorithm the opposite is done: the pellet parameters are estimated using the measured characteristics of the shadow. The pellet shadow is also characterised by three parameters: area $(A)$, largest $(D)$ and smallest linear dimension $(L)$ - see Fig. 5.
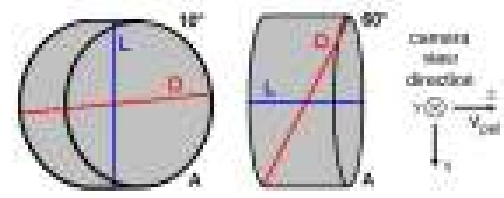

Figure 5 - Illustration of the pellet shadow parameters

For a given pellet size and orientation the shadow parameters are exactly determined. However, the inverse dependency is not unambiguous, that is, for a given $(D, L, A)$ a small set of $(r, h, \alpha)$ is possible. Therefore, the Bayesian method was applied to provide the probability distribution function (PDF) of the pellet parameters for a given $(D, L, A)$ triplet. This also allows the estimation of the uncertainty of the pellet parameters.

The complete process to reconstruct the pellet volume using a single-view shadowgraphy diagnostic is described in details in Ref. 15, therefore only a short overview will be given here. The basis of the Bayesian method is Bayes' Theorem, stating:

$$
P(X \mid Y)=\frac{P(Y \mid X) \cdot P(X)}{P(Y)}
$$

where $\mathrm{P}(X \mid Y)$ is the conditional probability of $X$ when $Y$ is true. This can be extended to arbitrary dimensions, in our case three, by substituting $X=(R, H, \alpha)$ and $Y=(d, l, a)$. Here, capital letters denote random variables whereas lowercase 
letters denote real (measured quantities). $P(Y)$ in the denominator can be expressed as $\int P(Y \mid X) \cdot P(X)$, so Bayes' theorem in the shadowgraphy case can be written as:

$$
P(R, H, \alpha \mid d, l, a)=\frac{P(d, l, a \mid R, H, \alpha) \cdot P(R, H, \alpha)}{\iiint_{R, H, \alpha} P(d, l, a \mid R, H, \alpha) \cdot P(R, H, \alpha)}
$$

where $P(R, H, \alpha \mid d, l, a) \equiv P(R, H, \alpha \mid D=d, L=l, A=a)$, called the posterior distribution, is the outcome of the calculation. $P(R, H, \alpha)$ is the so-called prior, which includes our knowledge about the result before the measurement. In the evaluation, we use a prior $P(R, H, \alpha)=P(R, H) \times P(\alpha)=$ $1 \times \cos (\alpha)$, which means that we expect the pellets to be of any size with isotropic 3D orientation ("flat prior"); the term $P(d, l, a \mid R, H, \alpha)$, called the likelihood, is our knowledge about the measurement. In the shadowgraphy case this is a function, resulting in a probability distribution of shadow parameters for a given combination of pellet parameters. The likelihood can be evaluated independently for any measurement. Using Eq. (6) one can calculate the PDF of the pellet volume in two steps:

$$
\begin{aligned}
& P(R, H \mid d, l, a)=\int_{\alpha} P(R, H, \alpha \mid d, l, a) \\
& P(V(R, H) \mid d, l, a)=V(R, H) \cdot P(R, H \mid d, l, a)
\end{aligned}
$$

where $V(R, H)$ is the volume of a cylinder with radius $R$ and height $H$. Using this, the reconstructed volume is calculated as the expected value, and the uncertainty as the standard deviation of the distribution:

$$
\begin{gathered}
V_{\text {pellet }}^{\text {rec }}=E(V)=\iint_{R, H} V(R, H) \cdot P(V(R, H) \mid d, j, a) \\
\sigma_{V}^{2}=E\left(V^{2}\right)-E^{2}(V)
\end{gathered}
$$

For a single-view shadowgraphy diagnostic the problem can be considered solved by this calculation. However, another view in the system can be regarded as another measurement of the same quantity. Therefore it can be used to improve the result. The same reconstruction algorithm (Equations (6)-(10)) is particularised for the second view as well, with the significant difference that instead of a flat prior in Eq. (6) the posterior distribution $P(R, H, \alpha \mid d, l, a)$ from the first view is used as a prior for the second view. The rest is calculated in the same way, but then this result will be based on both measurements.

The dual-view reconstruction algorithm has been tested against the single-view method in the following way: for both views the single-view algorithm has been implemented separately, resulting in reconstructed pellet volumes $V_{\mathrm{L}}$ and $V_{\mathrm{R}}$ for the "left" and "right" views, respectively. Also, the uncertainties $\sigma_{\mathrm{L}}$ and $\sigma_{\mathrm{R}}$ have been calculated. Then the dual-view algorithm has been performed, providing $V_{\text {dual }}$ and $\sigma_{\text {dual }}$. These values have been calculated and compared for a series of small (2.2 $\mathrm{mm} 3)$ and large $(35 \mathrm{~mm} 3)$ pellets in the following JET discharges" \#79565 (small) as well as \#79572,\#79573 and
\#79578 (large).The results are shown in Fig. 6. It can be seen that the dual-view algorithm provides a better estimate: the reconstructed volumes are in the same range, however, the uncertainties are about $10 \%$ smaller than in the single-view cases. It can also be seen that the relative error is significantly higher for small pellets.
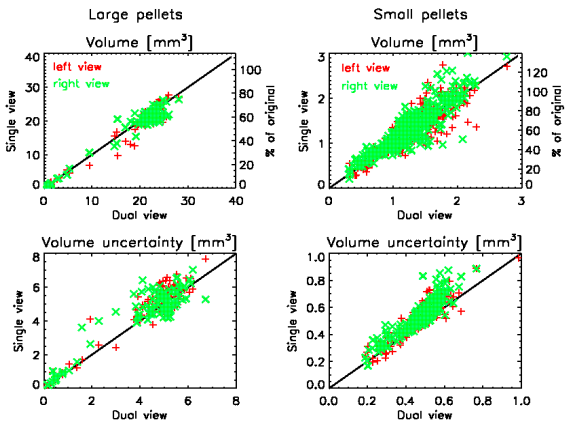

Figure 6 - Results of the Bayesian volume reconstruction methods. Single and dual-view algorithms are compared.

\section{Conclusion}

Two image processing techniques have been applied for the determination of two parameters important for pellet injection. Optimization of multi-resolution coarse-to-fine procedure and also the implementation of object tracking and detection allow fast optic flow image processing to determine ice extrusion velocity. Implementation of dualview shadowgraphy provides improved estimation of deuterium pellets volume by Bayesian analysis. The methods have been highly optimized and are able to provide fully automatic analysis. Validation has been performed on JET representative data.

\section{Acknowledgments}

This work, supported by the European Communities under the contract of Association between EURATOM and MEdC, IPP, ENEA, RMI KFKI, CIEMAT, was carried out under the framework of the European Fusion Development Agreement. The views and opinions expressed herein do not necessarily reflect those of the European Commission.

\section{References}

[1] S.L. Milora, et al., Nucl. Fusion 35657 (1995).

[2] H.W.Muller, et al., Phys. Rev. Lett. 832199 (1999).

[3] P.T. Lang, et al., Phys. Rev. Lett. 791487 (1997).

[4] P.T. Lang, et al., Nucl. Fusion 361531 (1996).

[5] P.T. Lang, et al., Fusion Eng. Des. 821073 (2007).

[6] T. Craciunescu, et al., J. . Nucl. Mater. 400205 (2010).

[7] A. Geraud et al., Fusion Eng. Des. 822183 (2007)

[8] B. Lucas, et al., Proc. Seventh International Joint Conference on Artificial Intelligence, Vancouver, Canada, 1981, p. 6749.

[9] A. Bruhn, et al., Int. J. Comput. Vision, 61211 (2005).

[10] B. Horn, et al., Artificial Intelligence, 17185 (1981).

[11] J. Bigün, et al., IEEE Trans. Pattern Anal. Mach. Intell. 13 775 (1991).

[12] A. Murari, et al., EFDA-JET-PR(10)03, submitted to IEEE Trans. Intell. Syst.

[13] G.W.Zack, et al., J Histochem Cytochem 25741 (1977).

[14] L.K. Huang, et al., Pattern Recogn. 2841 (1995).

[15] T. Szepesi, et al., Rev. Sci. Instrum. 79033501 (2008). 